\title{
The Significance of Computer Modeling and 3D Printing Technology for Facial Ectoprosthetics*
}

\author{
Svetlana Cherebylo ${ }^{1[0000-0003-0502-9681]}$, Vyacheslav Vnuk ${ }^{1[0000-0003-2536-6626]}$, \\ Evgeniy Ippolitov 1[0000-0002-0622-6727], Mikhail Novikov 1[0000-0003-0626-793X], \\ Pavel Mitroshenkov 2[0000-0002-3315-3973], Petr Mitroshenkov 2[0000-0002-7956-425X] \\ ${ }^{1}$ Institute on Laser and Information Technologies RAS - Branch of the Federal Scientific Re- \\ search Centre "Crystallography and Photonics" of RAS, Shatura, Russia \\ 2 FSBI "Clinical hospital №1” (Volynskaya) of the Russian President Administration, Moskow, \\ Russia \\ novikov@rambler.ru
}

\begin{abstract}
The integration of information technologies in healthcare practice significantly changes the methods of diagnosis and treatment, the forms of interaction of doctors with patients and colleagues, the organization of treatment and recovery of health. The field of reconstruction of the auricle is still a huge challenge for facial plastic surgeons and requires at various techniques to find the best treatment for each patient. The paper describes the application of computer modeling and laser stereolithography technology in surgical practice for auricular surfaces ectoprosthetics. To improve the accuracy and quality of the surgical intervention the positioning of external prosthesis is applied with the aid of personal templates and computer navigation. The accuracy of ectoprosthesis positioning when using a plastic mask template was $0.3-0.4 \mathrm{~mm}$, while computer navigation was $0.1-0.2 \mathrm{~mm}$. Using personalized templates is a simpler and more intuitive way of positioning that does not require expensive computer navigation systems. This example of ectoprosthetics shows the possibilities of various reconstructions of facial organs, not only the ear, but also, for example, the nose, using computer modeling and $3 \mathrm{~d}$ printing technologies
\end{abstract}

Keywords: Computed Tomography, Digital Model, Computer Modeling, Additive Technologies, Reconstructive Surgery.

\section{$1 \quad$ Introduction}

The integration of information technologies in healthcare practice significantly changes the methods of diagnosis and treatment, the forms of interaction of doctors

Copyright (C) 2020 for this paper by its authors. Use permitted under Creative Commons License Attribution 4.0 International (CC BY 4.0).

* This work was done with the financial support of the RFBR (Grant MK \# 18-29-03238). Work on laser stereolithography is executed at financial support of the Ministry of science and higher education (State task FSRC "Crystallography and Photonics" RAS). 


\section{S. Cherebylo et al.}

with patients and colleagues, the organization of treatment and recovery of health. The field of ear reconstruction is still a huge challenge for facial plastic surgeons and requires at various techniques to find the best treatment for each patient. Defects and deformities of the ear include not only acquired defects associated with trauma, burns, tumors, puncture defects, scars, and inflammation/allergies, but also congenital malformations of the ear, ranging from grade I malformations (such as prominent ears) to grade III malformations, including severe microtia changes. Depending on the defect and the surrounding circumstances, restoring a full ear is always the main goal of the patient and the plastic surgeon. In work [1], a fairly complete literary review of the application of modern methods of ear restoration is presented. Surgical practice has already acquired sufficient experience in using computer modeling and laser stereolithography for planning reconstructive operations [2-3] to successfully apply them to the treatment of patients.

The development of modern three-dimensional modeling and the introduction of a new generation of spiral computed tomography significantly expanded the possibilities of using information technologies in reconstructive surgery [4-8]. Specialized software allows to quickly process the graphical data of the patient's examination and create the digital three-dimensional models of any defect or pathology zone.

Digital models are increasingly used in the preparation and planning of surgery in maxillofacial surgery. They can be directly employed to create copies of pathologies and defects of a specific patient using additive technologies [2-4]. Personalized plastic copies of anatomical structures allow for effective pre-planning of complex operations. Based on digital models of defects or pathology zones of specific human diseases, can create unified databases for intelligent medical decision support systems. In most studies [4-7], the data from a patient's tomographic examination are used to create digital models. In [8-10], the photogrammetry method was applied to transform photographic images into a three-dimensional model. The analysis of the research shows that to create the most complete anatomical shape of the ear, especially with an internal structure, it is preferable to use the graphical data of the patient's tomographic examination.

The appearance of a man is primarily his face, and quite often there are situations when a person as a result of mechanical injury, serious illness or birth defects suffers from serious deformations of the face or from complete or partial absence of some of its organs. Such defects of the maxillofacial area are the cause of aesthetic discomfort, but modern medicine can solve this problem by performing reconstructive surgery to install individual ectoprostheses created using 3D printing technologies, which allow you to reproduce the anatomical model of a defective or lost organ with maximum accuracy. 3D printing technology has found its place in various branches of medicine, since its main advantage is the ability to create personalized models of various human organs and structures $[4,11,12]$. A breakthrough technology in the field of reconstructive surgery and implantology can be 3D-bioprinting - direct manufacturing of implants and organs of the required shape and functionality from biomaterials. A review of the current state of 3D-bioprinting shows that currently there are mainly experimental works on the search for effective biomaterials and synthesis processes, in practical surgery, bioprinting has not yet found direct application [13-16]. 


\section{Creating digital models and a virtual project}

The auricle has a complex anatomical structure, and to create an individual ectoprosthetics at the initial stage, it is necessary to have the most accurate three-dimensional representation of the anatomical changes of both bone and soft structures of the face. The initial data for building a personal three-dimensional model of the patient are the graphical data of the study on a computer tomograph [4].

To create virtual models, specialized software was used that allows to convert a set of gray images into a three-dimensional model. To do this, the image is segmented [17], and a certain threshold value is set for graying the image, which permits to separate bone fragments and soft tissues [18]. This process is very important, because it largely determines the accuracy and quality of the resulting digital models. STL models and specialized Magics software from Materialise, Belgium, were used for computer modeling in this work. To restore the model of the reconstructed auricle, the symmetry of the auricles was can use and a mirror image of the healthy zone was applied (see Fig. 1).
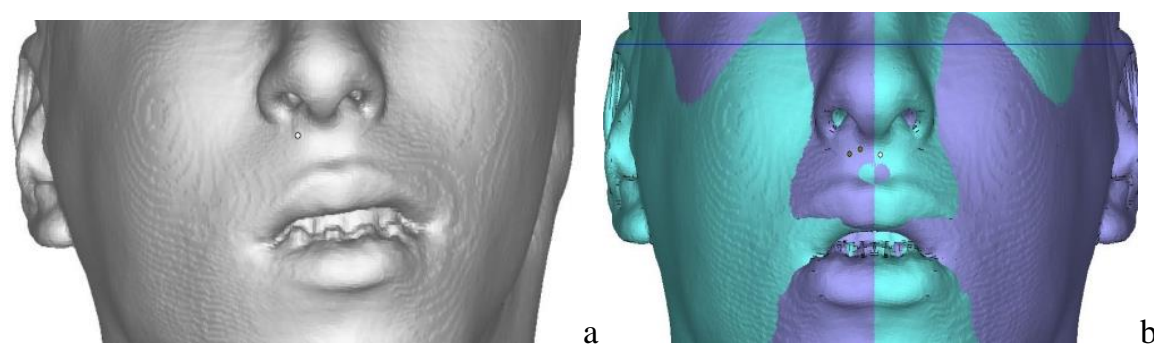

Fig. 1. Virtual project of soft tissue with the defect (a) and a mirror model (b).

Further work with the displayed auricle includes careful positioning of it. The three-dimensional model must be installed with regard for all the anatomical nuances, observing the proportions and symmetry. A number of software functions are used for this, namely rotation, displacement, merger, subtraction, smoothing, etc (see Fig. 2).
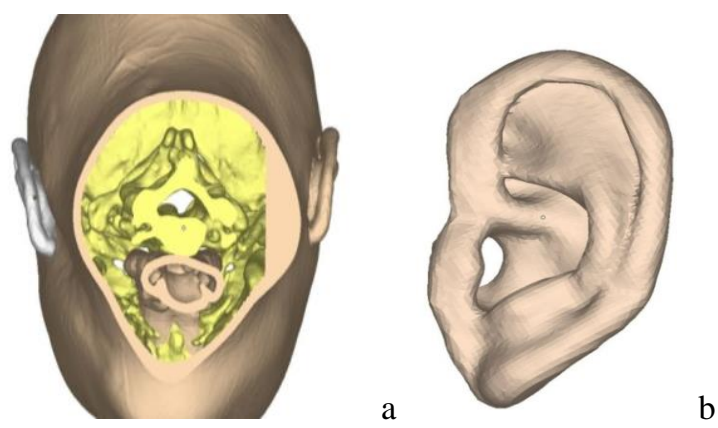

Fig.2 Positioning of the 3D model (a) of ear reconstruction (b). 
4 S. Cherebylo et al.

At the next stage of modeling, a model of template is created for the installation of extraoral implants, i.e. the fixing points of the support beam structure for the installation of the ectoprosthetics (see Fig. 3).

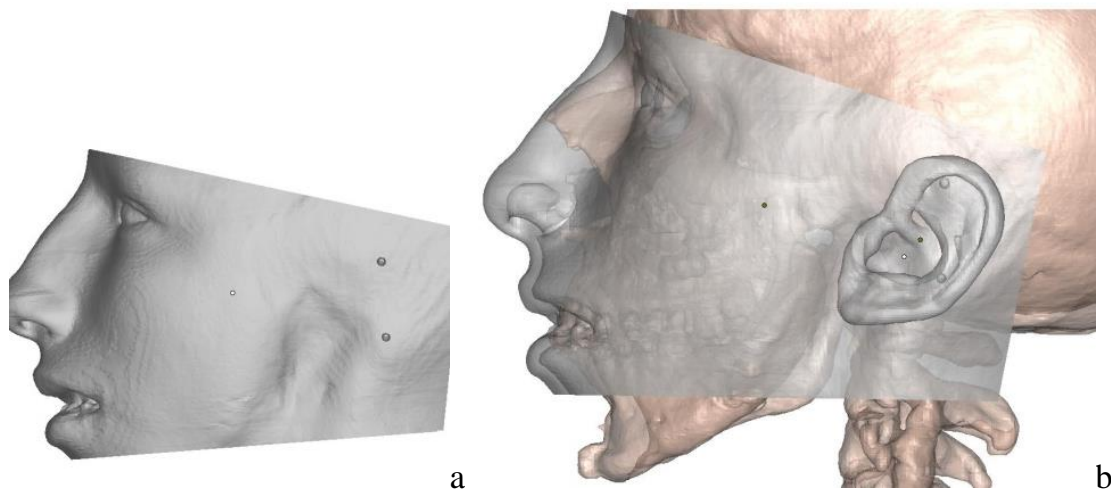

Fig.3 Virtual project of positioning of the 3D model (a) for reconstruction (b).

To use the navigation systems during the operation, a virtual project was developed (see Fig.4) for combining a digital model of the patient's facial skeleton with a designed mask template for positioning the ectoprosthetics fixation elements.

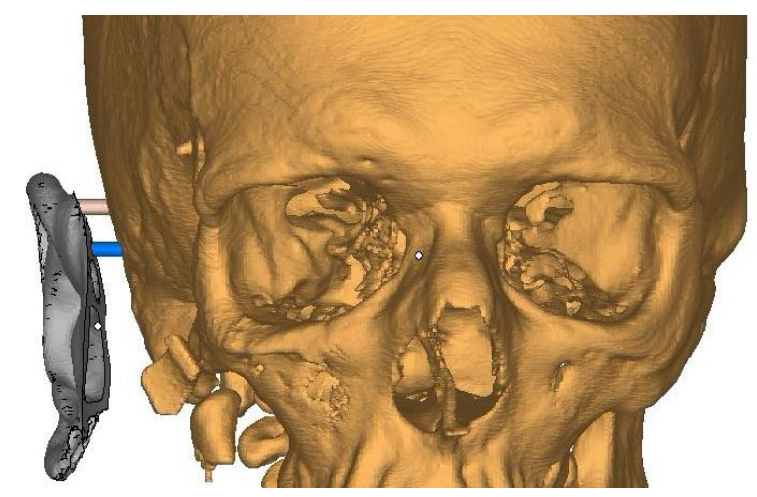

Fig.4. Virtual project of operation planning.

\section{Intraoperative computer navigation}

One of the main trends in clinical medicine is the intraoperative use of high-precision computer navigation, which allows improving patient's safety during surgery, increasing the accuracy of surgical interventions $[19,20]$. The widespread use of microsurgical equipment and high requirements for the accuracy of the intervention have led to the fact that even the best surgeons have almost reached the limit of their physical 
capabilities. The use of multispiral computed tomography (MSCT) significantly improved the 3-dimensional localization of the pathological process and increased the accuracy of surgical intervention and development of new imaging techniques, including an intraoperative one. The navigation system tracks the position of the instrument in the surgeon's hand and shows on the monitor how this instrument is positioned relative to the three-dimensional model of the patient created from preoperative tomographic examinations. Navigation allows to simulate the course of the operation on the three-dimensional reconstruction of the patient before performing surgical manipulations.

The problem of eliminating deformations of the facial skeleton of acquired and innate origin remains relevant to the present time. Complex spatial and geometric relationships between the facial skeleton and the contour of the soft tissues of the face contribute to the formation of combined deformations and defects of the facial skeleton when it is damaged or abnormally developed. The use of the standard technique of "template surgery" and individualization of the maxillofacial fixing and reconstructive implants, which in some clinical cases perform the function of a template for the reposition of bone fragments, is not always sufficient the possibility of achieving maximum accuracy of implant positioning. Currently, in order to improve the objectification and accuracy of intraoperative positioning of facial skeleton fragments, the method of computer navigation using modern surgical navigation stations is successfully applied.

The aim of the research was to develop an algorithm for applying the method of virtual planning of surgical interventions and intraoperative computer navigation to eliminate asymmetric deformities of the facial skeleton, to make a comparative analysis of various methods of registering the patient's head when performing intraoperative navigation.

All the patients underwent MSCT of the facial skeleton at the stage of preoperative examination. Virtual planning of the operation was performed by the external software Magics from Materialise, Belgium with the creation of a three-dimensional model that was imported into the navigation station before the operation. Intraoperative monitoring of the position of individual maxillofacial implants was achieved by the STRYKER computer navigation station (USA) (see Fig.5).

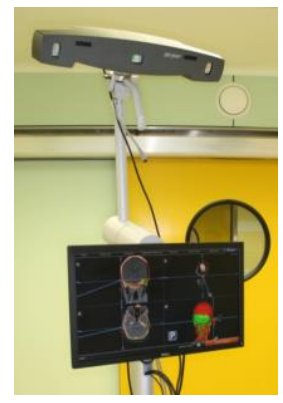

Fig.5. External view of the navigation station. 
6 S. Cherebylo et al.

Registration of the patient's head was performed in two ways: by the invasive and non-invasive contact methods. During the invasive registration method, the registration pins were installed in the patient's skull in the projection of the Nasion (N) point, the zygomatic sutures, and at the base of the Spina Nasalis Anterior (SNA). Performing registration by the non-invasive contact method did not require pre-installation of registration pins. The projections of points in the $\mathrm{N}$ region, the tip of the nose, and the medial and lateral corners of the eyes were used as registration points. To correct the registration error at the final stage of the procedure, additional registration points were collected from the patient's forehead using a contact or non-contact method, i.e. with a pointer or laser pointer. The external contour of displaced bone fragments and implants on the virtual model of the facial skeleton was used as reference points for eliminating post-traumatic deformations of the middle zone of the face. The positioning of implants in the postoperative period was monitored by combining a virtual preoperative model with postoperative MSCT data imported into the navigation station.

The analysis of the results of this study shows that the algorithm of intraoperative navigation allows achieving high accuracy of positioning of maxillofacial and extraoral implants. In this case, the accuracy of intraoperative control depends largely on the accuracy of the patient's head registration procedure. So, according to our research, the most accurate is the invasive method of registration, involving the use of bone pins. The registration error was $0.2-0.3 \mathrm{~mm}$. However, this method has a significant drawback - the need for preliminary hospitalization of the patient for the purpose of installing pins and performing MSCT. Only after this procedure, it is possible to perform preoperative virtual simulation, which significantly increases the duration of preoperative preparation. The non-invasive method of registering the patient's head does not require pre-installation of pins, but the accuracy of this method, according to our data, was lower, and the registration error was $0.6-0.7 \mathrm{~mm}$. This error can be compensated by collecting additional registration points from the skin surface of the patient's forehead, which is provided for by the registration Protocol of navigation stations. The combination of the preoperative virtual model and the results of the control MSCT of the facial skeleton using the image overlay method in the navigation station revealed an almost complete coincidence of the error in positioning of the osteotomized fragments and implants by the reference points with the data on the error in positioning of the registration points during the registration procedure of the patient's head, which indicates the fundamental importance of this stage in the process of intraoperative computer navigation. At the same time, the duration of the registration procedure did not significantly affect the overall duration of the surgical intervention and when mastering sufficient skills in performing this stage of navigation, it averaged 60 seconds. In order to achieve the maximum accuracy of modeling standard reconstructive implants, it is advisable to produce a stereolithographic model of the mold for the final packing of a standard reconstructive implant using a stereolithographic model of the patient's skull during preoperative preparation. Fig. 6 presents a clinical example of employment of intraoperative navigation when installing extraoral implants to fix the ear ectoprosthetics. 
The Significance of Computer Modeling and 3D Printing Technology... 7

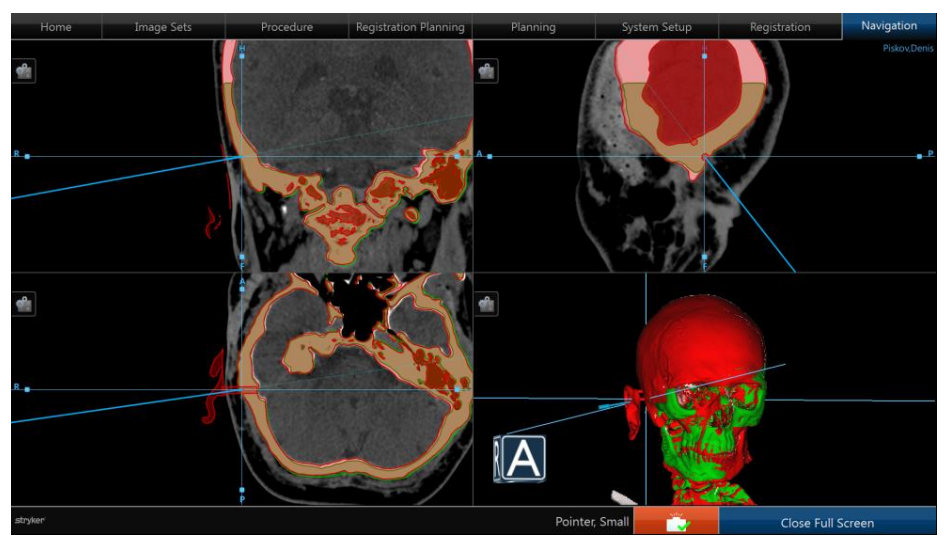

Fig.6 The use of intraoperative navigation during the installation of extraoral implants.

The model created as a result of virtual planning (see Fig.6) with extraoral implants placed on it was imported to the Stryker navigation station. The positioning of bone channels for implant placement was performed under the navigation control using a navigation pointer. The final check of the implant position was also performed using a pointer, as shown in Fig. 7.
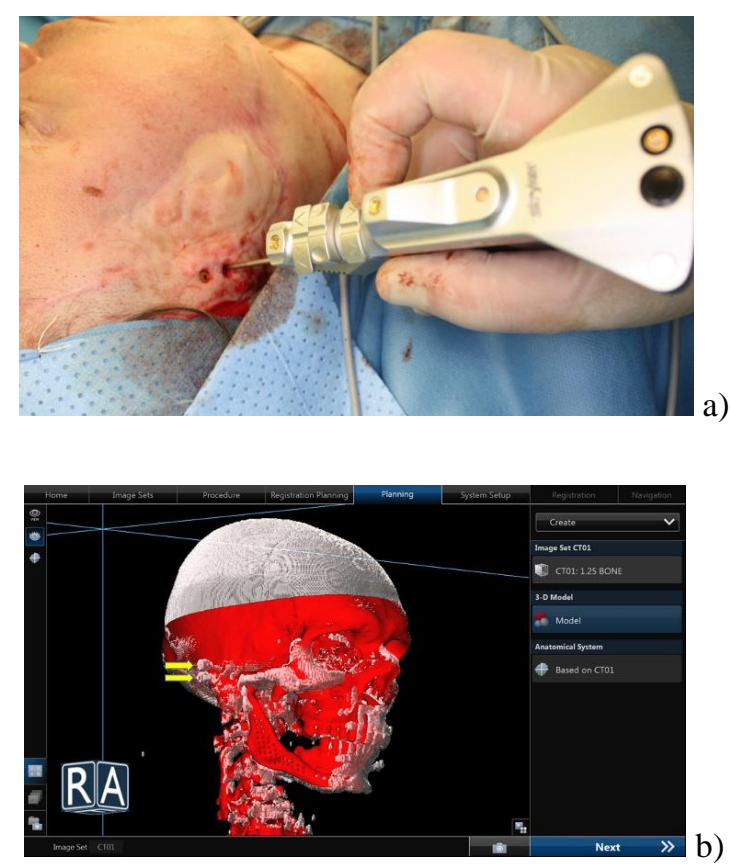

Fig. 7. The stage of combining the virtual and postoperative models (the arrows indicate the position of the implants); the position of the tip of the pointer (a) on the virtual model in on-line mode (b). 
In the postoperative period, when on combining the virtual model and the postoperative model of the patient's skull, the final control of the position of the extraoral implants was exerted, as shown in Fig. $7 \mathrm{~b}$ (the position of the implants is indicated by the arrows).

\section{$4 \quad$ Manufacturer of plastic models and their application}

Preliminary analysis of additive manufacturing methods (FDM, SLS, SLA) shows that laser stereolithography is the most promising technology for the production of personal templates in terms of accuracy and purity of surface reproduction, ease of processing and removal of residual material. To solve the problem of rapid production of three-dimensional objects of complex shape based on their computer models for medical applications, the LS150 laser stereolithography unit was developed at ILIT RAS. Plastic models were made by laser stereolithography, which creates real objects of complex shapes from a liquid photopolymer composition (see Fig. 8). The essence of the process is that the liquid resin is cured layer by layer in a given geometric plane under the influence of laser radiation [4].

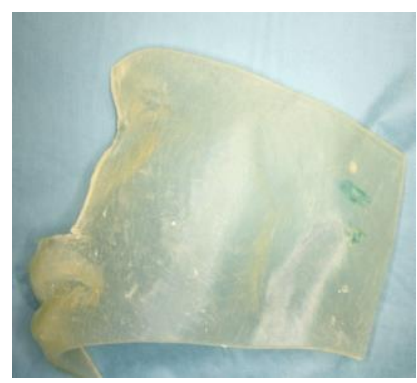

Fig.8 Plastic template of the mask model made by laser stereolithography.

The templates made by laser stereolithography was used during the operation for positioning and installing ectoprosthesis fixation elements (see Fig. 9).
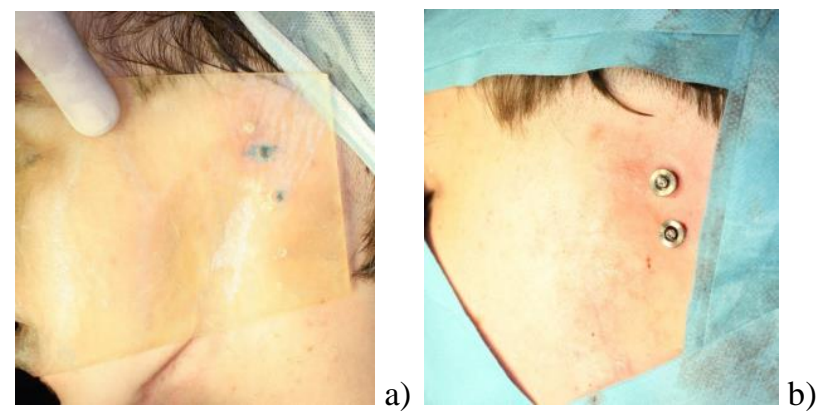

Fig.9. Application of plastic models when planning the installation (a) of an ectoprosthetics (b). 
The ectoprosthetics itself was made of medical silicone by casting a plastic prototype. The silicone construction is absolutely identical to the skin color and feels very similar to soft tissues (see Fig. 10).

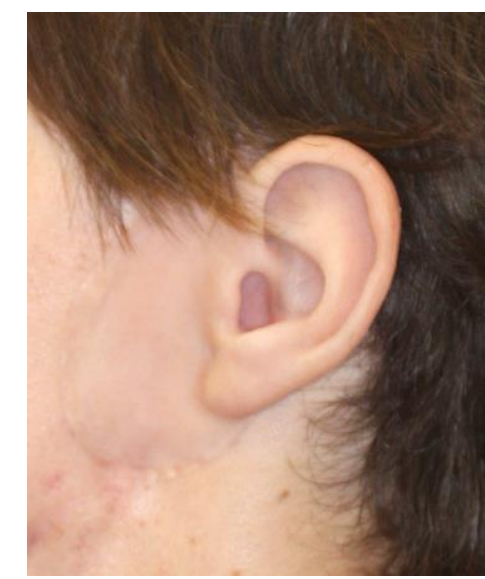

Fig. 10. The result of the ectoprosthetics installation

\section{Conclusion}

In the presented clinical cases it has become possible, thanks to computer modeling, the technology of laser stereolithography and computer navigation, to achieve full recovery of the body with the proper proportions and facial aesthetics, as well as to precisely position the clamping elements of ectoprosthetics (Fig.7, 10). The accuracy of ectoprosthesis positioning when using a plastic mask template was $0.3-0.4 \mathrm{~mm}$, while computer navigation was $0.1-0.2 \mathrm{~mm}$. Using personalized templates is a simpler and more intuitive way of positioning that does not require expensive computer navigation systems. This example of ectoprosthetics shows the possibilities of various reconstructions of facial organs, not only the ear, but also, for example, the nose, using computer modeling and $3 \mathrm{~d}$ printing technologies. Such surgical operations help to solve the problem of aesthetic disorders and as a result allow a person to restore psychological balance, socialize and gain self-confidence.

\section{Acknowledgments}

This work was done with the financial support of the RFBR (Grant MK \# 18-2903238). Work on laser stereolithography is executed at financial support of the Ministry of science and higher education (State task FSRC "Crystallography and Photonics" RAS). 
$10 \mathrm{~S}$. Cherebylo et al.

\section{References}

1. Storck, K.: Total Reconstruction of the Auricle: Our Experiences on Indications and Recent Techniques. BioMed Research International 2014, ID 373286, 15 p. (2014).

2. Stuchilov, V. A.: Clinical aspects of the use of laser stereolithography in the surgical treatment of injuries of the middle zone of the face. Clinical dentistry 3, 54-57 (RUS) (2001).

3. Naumann, A.: Clinical aspects and strategy for biomaterial engineering of an auricle based on three-dimensional stereolithography. European Archives of Oto-Rhino-Laryngology 260, 568-575 (2003).

4. Panchenko, V.Ya.: «Modern laser and information technologies», red., Interkontakt Nauka, Moscow, (RUS) (2014).

5. Mitroshenkov, P. N.: Reconstructive surgery of total and Subtotal defects of the upper, middle and lower zones of the facial skeleton. Synthesis of Books, Saint Petersburg, (RUS) (2010).

6. Zeng, W.: Fused deposition modelling of an auricle framework for microtia reconstruction based on CT images. Rapid Prototyping Journal 14(5), 280-284 (2008).

7. Guang-Ye, W.: Computer-assisted virtual preoperative planning in orthopedic surgery for acetabular fractures based on actual computed tomography data. Computer Assisted Surgery 21(1), 160-165 (2016).

8. Chen, Z.: Precision of three-dimensional stereo-photogrammetry $\left(3 \mathrm{dMD}^{\mathrm{TM}}\right)$ in anthropometry of the auricle and its application in microtia reconstruction. Journal of Plastic, Reconstructive and Aesthetic Surgery 68(5), 622-631 (2015).

9. Flores, R.: The technique for 3D printing patient-specific models for auricular reconstruction. Journal of Cranio-Maxillofacial Surgery 45(6), 937-943 (2017).

10. Zhou, J.: Three-dimensional autologous cartilage framework fabrication assisted by new additive manufactured ear-shaped templates for microtia reconstruction Journal of Plastic. Reconstructive and Aesthetic Surgery 69(10), 1436-1444 (2016).

11. Crafts, T.; Three-Dimensional Printing and Its Applications in Otorhinolaryngology- Otolaryngol. Head Neck Surg 156(6):999-1010 (2017).

12. Chae, Michael P.: Emerging applications of bedside 3D printing in plastic surgery. Frontiers in Surgery Volume 2| Article 25 (2015).

13. Guangdong, Z.: In Vitro Regeneration of Patient-specific Ear-shaped Cartilage and Its First Clinical Application for Auricular Reconstruction/ EBioMedicine 28, 287-302 (2018).

14. Kim, H.: Fabrication and characterization of 3D-printed elastic auricular scaffolds: A pilot study. Laryngoscope 129(2), 351-357 (2019).

15. Zachary, G.: Applications of 3-Dimensional Printing in Facial Plastic Surgery. J Oral Maxillofac Surg 74, 427-428 (2016).

16. Ji, Hyun Kim: Translation and Applications of Biofabrication. A. Ovsianikov . (eds.), 3D Printing and Biofabrication, Reference Series in Biomedical Engineering. Springer International Publishing AG (2016).

17. Musatyan, S.A.: Sposoby segmentacii medicinskih izobrazhenij. Trudy ISP RAN, 30(4), 183-194 (RUS) (2018).

18. Eijnatten, M.: The impact of manual threshold selection in medical additive manufacturing, J CARS 12, 607-615 (2017).

19. Park, Y.M.: Transoral robotic surgery for hypopharyngeal squamous cell carcinoma: 3Year oncologic and functional analysis. Oral Oncology 48, 560-566 (2012).

20. Lazzara. B.M.: Cyberknife radiosurgery in treating trigeminal neuralgia. J Neurointerv Surg. 5(1), 81-85 (2012). 